\title{
Integrating RTI service with primary health care
}

Population Council

Follow this and additional works at: https://knowledgecommons.popcouncil.org/departments_sbsr-rh

Part of the Demography, Population, and Ecology Commons, Family, Life Course, and Society Commons, International Public Health Commons, and the Women's Health Commons How does access to this work benefit you? Let us know!

\section{Recommended Citation}

"Integrating RTI service with primary health care," Asia and the Near East OR/TA Project Research Update. New Delhi: Population Council, 1998. 


\section{BACKGROUND}

The reproductive health movement worldwide has brought reproductive tract infections (RTI) under sharp focus as an urgent health need of women. RTIs include three types of infections: Sexually transmitted infections (STI), such as chlamydia, gonorrhoea, trichomoniasis, syphilis, chancroid, genital herpes, genital warts and human immunodeficiency virus (HIV) infection; endogenous infections, which are caused by overgrowth of organisms that can be present in the genital track of healthy women such as bacterial vaginosis and vulvovaginal candidiasis; and iatrogenic infections, which are associated with medical procedures such as assisted delivery and abortion under unhygienic conditions on insertion of IUD without proper asepsis care. While these infections are preventable or treatable, they are often the cause of infertility, ectopic pregnancy, cervical cancer, fetal loss, low birth weight infants, infant blindness and neonatal pneumonia.

The adverse health effects of RTIs, particularly STIs, is much higher on women than men. A factor which predisposes women to a higher level of morbidity than men is that in women an infection is often asymptomatic. This further delays treatment seeking. Table 1 gives the differential probabilities of transmission of infection from men to women, compared with women to men, and duration of infectivity once they get infected. In most of the developing world, including India, because of their lack of autonomy and negotiating power. women often have less control over their bodies and sexuality, less access to health care, and they often get poorer quality or delayed treatment in case of sickness.

Table 1: Differential Probability of Transmission of Infection from Men to Women Compared with Women to Men and Duration of Infectivity

\begin{tabular}{|c|c|c|c|c|c|c|}
\hline Parameter & Gonorrhoea & Syphilis & Chlamydia & Chancroid & $\begin{array}{r}\text { HIV without } \\
\text { ulcers }\end{array}$ & $\begin{array}{r}\text { HIV with } \\
\text { ulcers }\end{array}$ \\
\hline \multicolumn{7}{|c|}{ Transmission on probabilities } \\
\hline Male to female & 0.60 & 0.25 & 0.40 & 0.35 & 0.03 & 0.10 \\
\hline Female to male & 0.40 & 0.20 & 0.30 & 0.30 & 0.01 & 0.05 \\
\hline Average & 0.50 & 0.22 & 0.35 & 0.32 & 0.02 & 0.07 \\
\hline \multicolumn{7}{|c|}{ Duration of infectivity (days) } \\
\hline Male & 45 & 180 & 90 & 45 & 2880 & 2880 \\
\hline Female & 120 & 270 & 240 & 45 & 2880 & 2880 \\
\hline Average & 82 & 225 & 165 & 45 & 2880 & 2880 \\
\hline
\end{tabular}

Source: Mead Over \& Peter Piot (1993) in Dean T. Jamison et. al. Disease Control Priorities in Developing Countries. Oxford University Press (1993). 


\section{Population Council, India}

Thus women's health is doubly jeopardized: biologically, by exposing them to a higher risk of infection from their partners and longer duration of infectivity, as well as socially by subjecting them to a series of social restrictions and depriving them of their right to complete sexual health.

\section{Magnitude of RTI in India}

There are no precise statistics available, either at the national or state level, on the prevalence of RTIs in India. However, emerging evidence from community studies indicates that a significant proportion of Indian women suffer from RTIs, based on women's self report of symptoms, as well as clinical and laboratory examinations. For example, the Ford Foundation supported studies from four sites - urban slums in Bombay and Baroda, and rural areas of West Bengal and Gujarat. In all sites women reported symptoms indicative of RTI, such as excessive discharge ( 22 to 54 percent), backache ( 5 to 30 percent) and lower abdominal pain ( 9 to 22 percent). Similarly, studies carried out by the Centre for Operations Research and Training (CORT) in various parts of the country including Bihar, Madhya Pradesh, Rajasthan, Gujarat and Delhi slums show that in all sites more than 40 percent of the women reported one or more symptoms of RTI. Both, the 1995 Baseline Survey carried out by the Population Council in Agra and the recent endline survey in Agra and Sitapur districts of Uttar Pradesh revealed that 55 to 70 percent of women report at least one RTI symptom. On average, each woman reported $2.8 \mathrm{RTI}$ related problems.

Findings from clinical examinations also reveal that cervicitis ( 8 to 40 percent), vaginitis (10 to 15 percent) and pelvic inflammatory disease (1 to 17 percent) were the prominent morbidities. Similarly, clinical and laboratory examinations of rural women in Karnataka revealed that up to 70 percent had vaginitis, cervicitis or PID. In terms of specific infections, the laboratory assessment indicated bacterial vaginosis (18.2 percent), candidiasis ( 5.2 percent), trichomoniasis ( 7.5 percent), chlamydia ( 0.5 percent), gonorrhoea ( 0.8 percent), syphilis ( 1.5 percent), and urinary tract infections (6.5 percent). Apart from the community studies, clinical and microbiological studies have also confirmed the wide prevalence of RTIs among Indian women (for detailed review and references, see Integrating RTI Services in Primary Health Care System, Population Council, 1998).

In the public health scrvices in India, diagnostic and therapeutic services for STDs are provided by about 350 special STD clinics and STD/Skin departments of about 160 Medical Colleges. The majority of the STD clinics are located in metropolitan cities or district headquarter towns. Most of the patients who go to STD clinics are men. Very few women are able to avail of these services, both because of their limited accessibility and the stigma attached to the disease. It is estimated that only about 10 percent of patients with STDs (irrespective of sex) seek assistance from public clinics, while the remaining 90 percent go to private providers of health services.

Recent reproductive health research has clearly demonstrated that RTIs and their sequelae are closely linked to other areas of health care like family planning, safe motherhood, child survival and HIV prevention (see Figure 1). Hence, each of them could significantly contribute to the reduction and control of RTIs. The recently launched $\mathrm{RCH}$ Programme fully recognizes these linkages and 
considers prevention and treatment of RTI as an integral component of the reproductive health programme. The challenge is how to integrate RTI/STI control and prevention into existing health initiatives such as family planning, women's health, child survival and HIV prevention to get a synergistic effect.

\section{Pilot Sites for RTI Services}

In two districts of Uttar Pradesh, Agra and Sitapur, SIFPSA and the district health authorities conducted operations research (OR) to strengthen the public health sector by improving access to

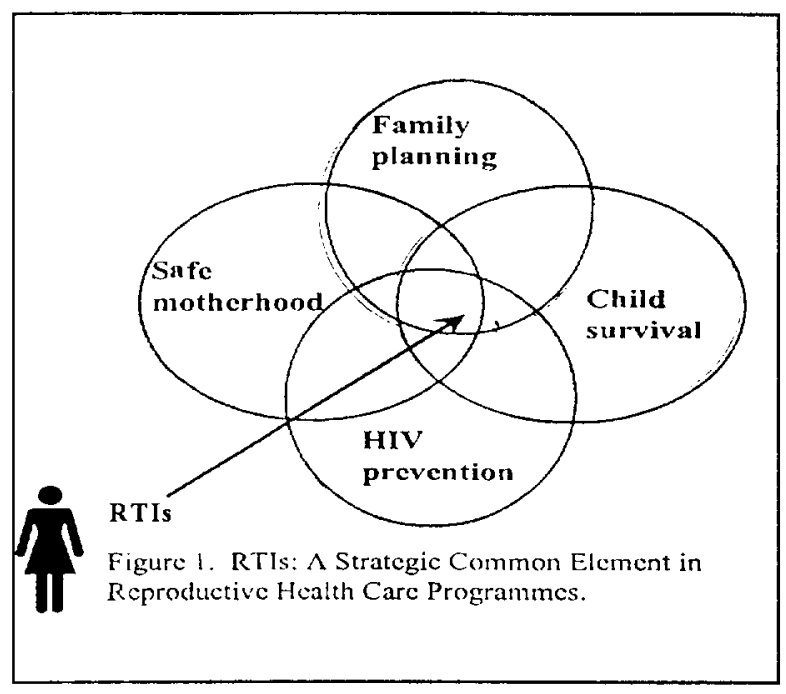
and quality of reproductive health services. The project has been funded by USAID and technical assistance for conducting the OR was provided by the Population Council. One project is testing the feasibility of integrating RTI services into the existing public health facilities. In rural Uttar Pradesh, most women object to a pelvic examination conducted by a male doctor. Only Post Partum Centres (PPCs) or Community Health Centres (CHCs) are considered appropriate, as at these health facilities generally a lady doctor is posted.

Accordingly Mehmoodabad PPC and Sidhauli CHC, which are located in the OR area of Sitapur district, were selected for the study. Mehmoodabad PPC covers a population of about 143,000, while Sidhauli CHC offers different health and family planning services to a population of about 146,000. In the OR area of Agra district, there was neither a PPC nor a CHC. None of the 3 Block PHCs covered have a lady gynaecologist. However, the PHC at Achhnera is covered by a special SIFPSA scheme in which private lady doctors are contracted to provide gynaecological services on fixed days. Considering the importance of the SIFPSA scheme, Achhnera was also included in the study. All three types of health facilities have laboratories which are equipped to conduct simple microscopic examination of blood, urine, and sputum for TB tests. Thus it was possible to upgrade the available laboratory facilities, and with the requisite training of a gynaecologist and the laboratory technician, it was felt that RTI services could be provided at these health facilities.

\section{Interventions}

The intervention consisted of several activities including: situation analysis and a subsequent upgradation of laboratory facilities at the study sites; training laboratory technicians to be able to diagnose selected RTI infections; training doctors in the syndromic approach for the management of RTI/STI cases, and the supply of essential laboratory reagents. Drugs for treatment were provided after a gap of 6 months from the initiation of the project to see how improved and regular availability of drugs improves the utilization of the clinics' services. Each of these aspects is briefly discussed below. 
Situation Analysis and Upgradation of Laboratories: The situation analysis of the laboratory facilities revealed that in all the three sites, essential equipment, i.e. microscope, centrifuge and burner, was available. Recurring supplies such as slides, cover slips, test tubes, syringes, needles and reagents were in short supply and were provided under the project. With these supplies, the laboratories were upgraded to carry out simple RTI diagnostic tests like saline wet mounts and gram stains.

Training of Laboratory Technicians: The situation analysis of laboratory facilities was followed by a 10 day training of laboratory technicians. Given that this approach will gradually be extended to all the Block PHCs and rural hospitals by the National STD/AIDS Control Programme (NACO), the laboratory training was extended to all the ten laboratory technicians attached to the facilities in the $O R$ areas of the districts. The training was organized at KG Medical College, Lucknow in collaboration with its Department of Microbiology, with the additional support of NACO and Population Council staff. Practical training was given more emphasis than theoretical orientation. The laboratory technicians were trained in laboratory techniques for identifying several infections, including candidiasis, trichomoniasis, bacterial vaginosis, chlamydia, and syphilis. They were also given training in general laboratory procedures such as sterilization of glassware, maintenance of equipment, quality control, registration and record keeping.

Training of Doctors in Syndromic Approach for the Management of RTI/STD Cases: A total of 15 doctors and two tutors from ANM Training Centres (one each from Agra and Sitapur) were given 4 days of training in the syndromic approach for the management of RTIs. The syndromic approach to RTI case management provides clinicians with flow charts for treatment for RTIs based on symptoms, clinical exam and risk characteristics. To keep the training compatible with what the MOH\&FW is implementing through the National STD/AIDS Control Programme, it was decided to use the same curriculum, guidelines and the six training modules developed by NACO for this purpose. The training was organized in collaboration with KG Medical College and NACO officials, and held at the State Institute of Health and Family Welfare. National resource persons used by NACO were invited to assist in the training. Practical orientation was given to the doctors at the Ob/Gyn Department of KG Medical College, where special arrangements were made to provide facilities for microscopic examination. The flow chart on syndromic management of RTI developed by NACO was reproduced and provided to the doctors to facilitate their work.

A comparison of pre and post training knowledge of the doctors showed significant improvements in knowledge of RTIs as well as the syndromic approach to RTI case management (see Integrating RTI Services in Primary Health Care System, Population Council, 1998).

Supply of Essential Drugs: Essential drugs to treat RTI patients were also supplied to the clinics. The delay in their arrival was due more to the lack of initiative on the part of one of the senior district officials than any administrative procedure. 


\section{Findings}

RTI services were provided, after training of Medical Officers, from April 1997. An analysis of data on the women who were provided with reproductive health services during the study period at the three study sites is presented in Table 2. As the results show, the PPCs were used extensively by women, and a total of 6905 women were served during the study period. Of these, 626 (9.1 percent) had RTI complaints. In the case of the Sidhauli CHC, due to the non-availability of a lady gynaecologist, only 1181 women were provided with services. Of these, 153 (13 percent) had RTI problems.

Table 2: Women attending reproductive health clinics and their problems

\begin{tabular}{|c|c|c|c|c|c|c|}
\hline \multirow[t]{2}{*}{ Problems** } & \multicolumn{2}{|c|}{$\begin{array}{l}\text { Mehmoodabad (6905) } \\
\text { Apr } 1997 \text { - Jan } 1998 \\
\end{array}$} & \multicolumn{2}{|c|}{$\begin{array}{c}\text { Sidhauli (1181) } \\
\text { Apr } 1997 \text { - Jan } 1998\end{array}$} & \multicolumn{2}{|c|}{$\begin{array}{c}\text { Achhnera (3233) } \\
\text { Nov } 95 \text { - Jan } 1998 \\
\end{array}$} \\
\hline & Nos. & $\% *$ & Nos. & $\% *$ & Nos. & $\% *$ \\
\hline Gynaecology & 1764 & 25.6 & 281 & 23.8 & 731 & 22.6 \\
\hline Dysfunctional uterine bleeding & 1289 & 18.7 & 4 & 0.3 & 201 & 6.2 \\
\hline CA-genital & 46 & 0.7 & 14 & 1.2 & 147 & 4.6 \\
\hline Dysmenorrhoea & 39 & 1). 6 & 9 & 0.8 & 16 & 0.5 \\
\hline Sterility & 195 & 2.8 & 15 & 1.3 & 206 & 6.4 \\
\hline Urinary tract infection/vaginitis & 29 & 1). 4 & 44 & 3.7 & 50 & 1.5 \\
\hline Amenorrhoea & 38 & 0.6 & 42 & 3.6 & 111 & 3.4 \\
\hline Others & 128 & 1.9 & 153 & 13.0 & - & - \\
\hline Obstetrics & 2726 & 39.5 & 363 & 30.7 & 1673 & 51.7 \\
\hline Antenatal care & 2081 & 311.1 & 309 & 26.2 & 1408 & 43.6 \\
\hline Postnatal care & 151 & 2.2 & 18 & 1.5 & 63 & 1.9 \\
\hline Delayed menses & 494 & 7.2 & 36 & 3.0 & 202 & 6.2 \\
\hline Abortion & 112 & 1.6 & 44 & 3.7 & 235 & 7.3 \\
\hline Complete & 6 & 1).1 & 6 & 0.5 & 168 & 5.2 \\
\hline Incomplete & 44 & i). 6 & 7 & 0.6 & 67 & 2.1 \\
\hline Risk of abortion & 62 & 0.9 & 31 & 2.6 & - & - \\
\hline RTI & 626 & 9.1 & 153 & 13 & 488 & 15.1 \\
\hline Vaginal discharge/Leucorrhea & 291 & 4.2 & 26 & 2.2 & 246 & 7.6 \\
\hline Lower abdominal pain with vaginal discharge & 49 & 0.7 & 73 & 6.2 & 15 & 0.5 \\
\hline PID & 286 & 4.2 & 54 & 4.6 & 227 & 7.0 \\
\hline Family Planning & 300 & 4.3 & 42 & 3.6 & 77 & 2.4 \\
\hline FP services & 210 & 3.0 & 24 & 2.1 & 6 & 0.2 \\
\hline Follow-up & 90 & 1.3 & 18 & 1.5 & 71 & 2.2 \\
\hline Others & 1377 & 19.9 & 298 & 25.2 & 340 & 10.5 \\
\hline
\end{tabular}

The Achhnera PHC, which has been covered under the SIFPSA's private lady doctor scheme since November 1995, provided general health services to 3233 women in 100 clinic days. Of these, 15.1 percent sought treatment for RTI problems. However, during the study period (April 1997 - January 1998) the number of women who were provided reproductive health services at the PHC was 1412 , and the proportion of RTI cases was 13.2 percent. Thus the study shows that between 9 and 15 percent of the women attending reproductive health clinics came with symptoms of one or more RTIs. Further analysis also revealed that PID appeared to be one of the most common diagnoses 
(see Table 2). Vaginal discharge was also a common symptom, which was reported in about half of women with RTI complaints.

The study also revealed that despite the availability of laboratory facilities, the doctors often depend more on their clinical examination and reported symptoms of the patient than on confirming the diagnosis with lab tests. As Table 3 shows, out of the total suspected RTI cases at Mehamoodabad PPC, in only about half of the cases was a lab test done. The proportion was one-fourth in Sidhauli, and nearly one sixth of the cases at Achhnera PHC were tested. About 7 percent of the cases, when tested in the laboratory, were positive.

Specific antibiotics recommended in the syndromic approach were not available at any of the clinics. Hence, most of the women were given prescriptions to purchase the drugs from the market. According to the doctors, most of the women did purchase the medicine and took the treatment as advised by the doctors. However, no follow-up was done for partner notification and treatment.

Table 3: Total number of suspected RTI cases, number of cases subject to laboratory tests and results obtained

\begin{tabular}{lccccc}
\hline & $\begin{array}{l}\text { Number of } \\
\text { suspected } \\
\text { RTI cases }\end{array}$ & $\begin{array}{l}\text { No. of lab. } \\
\text { tests done }\end{array}$ & $\begin{array}{l}\text { \% of RTI } \\
\text { cases subject } \\
\text { to test }\end{array}$ & $\begin{array}{l}\text { No. of positive } \\
\text { cases }\end{array}$ & $\begin{array}{l}\% \text { of Lab tests } \\
\text { with a positive } \\
\text { result for RTI }\end{array}$ \\
\hline Mehmoodabad & 626 & 327 & 52 & 25 & 8 \\
Sidhauli & 153 & 40 & 26 & 24 & 6 \\
Achhnera & 187 & 30 & 16 & - & - \\
\hline
\end{tabular}

\section{LESSONS LEARNED}

An analysis of the processes involved in implementation of the project and findings from the three sites suggest several important points.

- Most of the CHCs, PPCs and Block PHCs have laboratory facilities which, with marginal resource input, could be made functional for confirming the clinical findings of trichomoniasis, candidiasis and bacterial vaginosis. Quality control of laboratory procedures would have to be established to ensure appropriate standards are maintained.

- In most of these clinics, reagents to perform these tests (wet mount and gram staining) are not available. Hence, their regular supply is critical to keep these laboratories functional. A situation analysis of these laboratory facilities could be rapidly done using a check list.

- Generally the laboratory technicians are eager and capable of carrying out these tests. A five day reorientation of lab technicians as recommended by NACO may be sufficient. A rigorous 10 day training given under the present study is not required. Instead, a practical follow-up reorientation after 3 months may be more useful. 


\section{Population Council, Indiu}

The guidelines and manuals developed by NACO dre useful. The present study shows that 4 days training is sufficient and effective in imparting knowledge about the syndromic approach for the diagnosis and management of RTI to gynaecologists already treating clients with infections. The general practitioners (GP) may need a reorientation within 3 months after the first training in the syndromic approach. This needs to be tested and confirmed.

- Administrative delays in procuring the reagents and drugs, and frequent transfer or absence of gynaecologists posted at the health facilities are some of the serious bottlenecks in effective implementation of the service. For example, despite the fact that funds were provided under the project to purchase reagents, it took almost 4 months before they were procured and supplied to clinics. If, after training, the laboratory technicians do not get the opportunity to use their newly acquired skills, the impact of training is greatly reduced. Similarly, despite repeated reminders, essential drugs for the treatment of RTI were released by the CMOs only in January 1998.

Similarly at the CHC in Sidhauli, the gynaecologist who was trained under the project was transferred soon after training and the lady doctor who replaced her went on leave for a period of two months. The doctor who replaced her at the gynaecology clinic at the CHC was an experienced ophthalmologist, and had not received any training in the management of RTIs. Administrative difficulties could easily frustrate the initiatives taken for offering RH services at these health facilities.

Among the three types of participant health facilities, the PPCs seems to be the most appropriate. As PPCs are generally attached to a women's hospital, a much larger number of women (6905 during April 1997 - January 1998) avail of their services. Consequently, the probability of finding RTI cases and putting laboratory facilities to use is also significantly greater. A discussion with the laboratory technicians at the PPCs revealed that they were quite happy with the added responsibility of testing for RTIs. As they are now regularly doing these tests, it has become a normal task, and their diagnoses as reported by the doctor were quite helpful.

In contrast, at the Sidhauli $\mathrm{CHC}$, the frequent transfer and absence of trained lady doctors led to poorer case detection and treatment. This is evident from the fact that only 5 cases in April, and 7 cases in September were sent for lab testing. A lady doctor who was visiting the CHC under the SIFPSA scheme often diverted RTI patients to her own private clinic at Sidhauli or Lucknow. Since September, she has been shifted to another PHC (Kamlapur) as the doctor on leave has returned. Such administrative weaknesses contribute significantly to the under-utilization of public health facilities. Drawing attention to these practices improved the situation, and more patients with suspected RTIs were tested between October 1997 and January 1998.

- PHCs, where only male doctors are posted, do not create enough demand for services from women suffering from RTIs or other major reproductive health problems. Generally, they go to nearby towns for treatment. However. at Achhnera PHC where a private female gynaecologist was hired to visit the clinic for 4 hours a week, on an average 32 women per day (ranging between 15-57 per clinic day) come to avail of the clinic's services. $\Lambda \mathrm{n}$ analysis 
of the patients' problems reveals that 15.1 percent came for RTI complaints. However, all of them were not provided with the required laboratory test. Staff responsibilities and delegation problems (like who will take the slides and deliver them to the laboratory as no ANM is attached to the OPD), and occasionally the non-availability of laboratory technicians reinforced the lady doctor's general habit of prescribing treatment on symptoms and/or clinical examination alone.

- At all the study sites, the medicines for the treatment of RTI patients are in short supply. Hence, patients generally are prescribed medicines for purchase from the market. The study shows that generally the patients did not object to purchasing the medicines.

- A separate cost study revealed that current budget for drugs is inadequate for even RTI services. See the POLICY BRIEF on RTI Case Management.

- There are a number of questions which this study could not answer.

Partner notification and treatment. Presently the social environment in which PPCs function does not encourage men to come with their spouses for consultation and treatment. The problem of partner tracing and ways of enhancing treatment of partners needs further study.

Since patients are ready to buy medicines in the market, will the provision of drugs at the clinics increase overall utilisation of the health facilities?

How can reproductive health services be expanded beyond PPCs, particularly at PHCs where no female doctors are posted? SIFPSA's private lady doctor scheme or reproductive health camps could be good alternatives. However, these schemes should be carefully planned, tested and documented for expansion to larger arcas.

Preventive education as well as messages for early identification and treatment of RTI requires that appropriate IEC efforts be tested. Involving male and female health workers to educate and inform community members, and youth in particular, should be developed and their impact on the target groups and the community should be assessed.

This UPDATE on the OR Project in Agra and Sitapur Districts in Uttar Pradesh was conducted with support from the Government of Uttar Pradesh, SIFPSA and the Population Council's Asia and Near East Operations Research and Technical Assistance Project. The ANE OR/TA Project is funded by the US Agency for International Development, Office of Population under Contract No. DPE-C-00-90-0002-10, Strategies for Improving Service Delivery. 